\title{
Experiments with Firefly Algorithm
}

\author{
Rogério B. Francisco ${ }^{1,2}$, M. Fernanda P. Costa $^{2}$, Ana Maria A. C. Rocha ${ }^{3}$ \\ ${ }^{1}$ Escola Superior de Tecnologia e Gestão de Felgueiras, 4610-156 Felgueiras, Portugal \\ rbf@estgf.ipp.pt \\ ${ }^{2}$ Centre of Mathematics, University of Minho, 4710-057 Braga, Portugal \\ mfcemath. uminho.pt \\ ${ }^{3}$ Algoritmi Research Centre, University of Minho, , 4710-057 Braga, Portugal \\ arocha@dps. uminho.pt
}

\begin{abstract}
Firefly Algorithm (FA) is one of the recent swarm intelligence methods developed by Xin-She Yang in 2008 [12]. FA is a stochastic, nature-inspired, metaheuristic algorithm that can be applied for solving the hardest optimization problems. The main goal of this paper is to analyze the influence of changing some parameters of the FA when solving bound constrained optimization problems. One of the most important aspects of this algorithm is how far is the distance between the points and the way they are drawn to the optimal solution. In this work, we aim to analyze other ways of calculating the distance between the points and also other functions to compute the attractiveness of fireflies.

To show the performance of the proposed modified FAs a set of 30 benchmark global optimization test problems are used. Preliminary experiments reveal that the obtained results are competitive when comparing with the original FA version.
\end{abstract}

Keywords: Firefly algorithm, Unconstrained Optimization.

\section{Introduction}

The main objective of the optimization methods is to determine the maximum or the minimum of mathematical functions, called objective functions, which may be, or may not be, subject to constraints on its variables. Due to the wide variety of practical applications, optimization algorithms have been increasingly studied in the area of engineering and applied mathematics. The optimization methods can be divided into two major groups: deterministic and stochastic methods, which may use or not the derivatives of the objective and constraint functions.

The most of deterministic methods are local search methods. These methods are characterized for producing always the same set of solutions (optimal points) if the algorithm start under the same initial conditions. In turn, the stochastic methods are characterized by having one or more components of randomness, called stochastic components. This implies that for the same problem, and subject to the same initial conditions, these algorithms may not generate the same optimal solutions. There is a 
range of possibilities of how to form this stochastic component. For example, one way is to make a simple randomization by randomly sampling the search space or by making random walks. The majority of this type of methods is considered as metaheuristic.

Firefly Algorithm (FA) is an algorithm that belongs to the second group, that is, a stochastic and metaheuristic algorithm, and it was developed by Yang [13, 14]. It is a recent nature inspired optimization algorithm, inspired by the social behavior of fireflies, and is based on their flashing and attraction characteristics.

The firefly algorithm is one example among many, of the so called bio-inspired algorithms. Among such methods, the Genetic Algorithms [7], the Particle Swarm Optimization [5], the Ant Colony Optimization [3], the Artificial Fish Swarm Algorithm [11], are the best known algorithms that are inspired by phenomena and/or behaviors of nature and the animal world.

The first studies on bio-inspired algorithms are due to Reynolds [10] and Heppner and Grenander [8], which are based on the behavior and movement of flocks of birds. The Ant Colony Optimization algorithm, developed by Dorigo et al. [4], is based on the social behavior of insects and their form of communication to find the optimal path between their colony and its power supply. The Particle Swarm Optimization based on the movement of flocks of birds is due to Eberhart and Kennedy [6].

Following the trend of the study of natural collective behavior, Yang [13] introduced a new algorithm, known as Firefly Algorithm, inspired by the collective behavior of fireflies, specifically in how they attract each other. Previous studies have demonstrated that the FA obtained good results, indicating its superiority over some bio-inspired methods $[9,12,14]$.

The paper is organized as follows. Section 2 briefly presents the key ideas of the original FA and describes the proposals to change attractiveness function in the FA. The numerical experiments are reported in Section 3 and the paper concludes in Section 4 .

\section{Firefly Algorithm for Bound Constrained Problems}

In this study we are interested in solving the bound constrained global optimization problem by FA. The mathematical formulation of the optimization problem to be addressed in this paper is stated as follows:

$$
\begin{array}{cc}
\min _{x \in \mathbb{R}^{n}} & f(x) \\
\text { subject to } & l \leq x \leq u
\end{array}
$$

where $f(x)$ is a continuous nonlinear objective function, $l$ and $u$ are the lower and upper bounds of the variables.

Following the firefly algorithm and the proposal changes to the attractiveness function are presented. 


\subsection{Original FA}

The firefly algorithm is based on three main principles:

1. All fireflies are unisex, implying that all the elements of a population can attract each other.

2. The attractiveness between fireflies is proportional to their brightness. The firefly with less bright will move towards the brighter one. If no one is brighter than a particular firefly, it moves randomly. Attractiveness is proportional to the brightness which decreases with increasing distance between fireflies.

3. The brightness or light intensity of a firefly is related with the type of function to be optimized. In practice, the brightness of each firefly can be directly proportional to the value of the objective function.

This algorithm is based on two key ideas: the light intensity emitted and the degree of attractiveness that is generated between two fireflies.

The light intensity of firefly $i, I_{i}$, depends on the intensity $I_{0}$ of light emitted by firefly $i$ and the distance $r$ between firefly $i$ and $j$. In [13], the light intensity $I_{i}$ varies with the distance $r_{i j}$ monotonically and exponentially. That is

$$
I_{i}=I_{0} e^{-\gamma r_{i j}}
$$

where $\gamma$ is the light absorption coefficient. In theory, $\gamma \in[0 ;+\infty[$, but in practice $\gamma$ can be taken as 1 .

Since the attractiveness $\beta_{i j}$ of the firefly $i$ depends on the light intensity seen by an adjacent firefly $j$ and its distance $r_{i j}$, then the attractiveness $\beta_{i j}$ is given by:

$$
\beta_{i j}=\beta_{0} e^{-\gamma r_{i j}{ }^{2}}
$$

where $\beta_{0}$ is the attractiveness at $r_{i j}=0$.

In the original method, the distance $r_{i j}$ between any two fireflies $i$ e $j$, at $x_{i}$ and $x_{j}$, could be given by the Cartesian distance (or 2-norm):

$$
r_{i j}=\left\|x_{i}-x_{j}\right\|_{2} .
$$

The movement of a firefly $i$ towards another brightest firefly $j$ is given by:

$$
x_{i}=x_{i}+\beta_{i j}\left(x_{j}-x_{i}\right)+\alpha \epsilon_{i j}
$$

where $\epsilon_{i j}$ is a random parameter generated by a uniform distribution and $\alpha$ is a parameter of scale.

In this paper, the light intensity of a firefly $i, I_{i}$, is determined by its objective function value.

The pseudo code of the Firefly Algorithm for bound constrained optimization problems can be summarized as follows. 


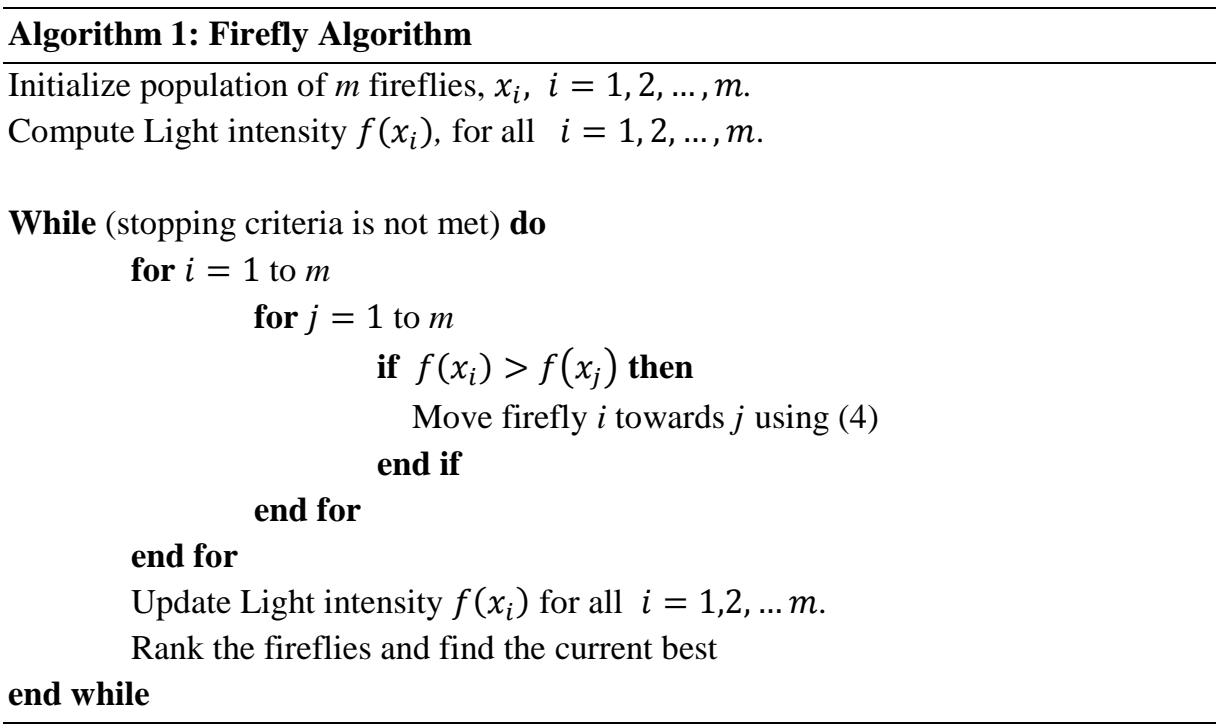

\subsection{Modified Attractiveness Approach}

In this paper we want to study the effect of changing the calculation of the attractiveness function $\beta$ (see (2)), either by changing the way of calculating the distance $r$ between two points, either by changing its algebraic expression.

Let $p$-norm of $x \in \mathbb{R}^{n}$, for $p \in \mathbb{N}$, be the norm defined by:

$$
\|x\|_{p}=\left(\sum_{i=1}^{n}\left|x_{i}\right|^{p}\right)^{\frac{1}{p}}
$$

Let the infinity-norm, $\|x\|_{\infty}$, be defined by:

$$
\|x\|_{\infty}=\max \left\{\left|x_{i}\right|\right\}_{i=1, \ldots, n} .
$$

In a first approach, a numerical experiment based on the change of the use of 2norm, to compute the distance between two fireflies, is analyzed (see (3)). In this experiment, the 1-norm, 4-norm, 10-norm and infinity-norm are considered. The goal is to investigate these modifications in order to improve the efficiency and robustness of the FA (see (2)).

In a second approach, two new attractiveness functions, $\beta^{1}$ and $\beta^{2}$, are considered. Thus, a new attractiveness function, $\beta^{1}$, that is monotonically decreasing as (2) is proposed:

$$
\beta_{i j}^{1}=\left\{\begin{array}{ccc}
\beta_{0} e^{-\gamma r_{i j}{ }^{2}} & \text { if } \quad r_{i j} \leq d \\
0.5 & \text { if } \quad r_{i j}>d
\end{array}\right.
$$


Here, for a given value of $d$, if the distance between two fireflies is lesser or equal than $d$, then $\beta^{1}$ exponentially decreases with the distance. Whenever the distance between two fireflies is greater than $d$, the attractiveness takes a constant value. The motivation for this approach is due to the fact that in (2) the value of attractiveness can be considered negligible for certain values of distance.

Finally, another attractiveness function that takes into account the average rate of change of the firefly brightness is considered. This function, denoted by $\beta^{2}$, is defined by:

$$
\beta_{i j}^{2}=\left\{\begin{array}{ccc}
\beta_{0} e^{-\frac{f\left(x_{i}\right)-f\left(x_{j}\right)}{r_{i j}}} & \text { if } & f\left(x_{i}\right)>f\left(x_{j}\right) \\
0 & \text { otherwise. }
\end{array}\right.
$$

\section{$3 \quad$ Numerical Experiments}

The FA algorithm was coded in Matlab and the computational tests were performed on a PC with a $2.53 \mathrm{GHz}$ Intel(R) Core (Tm) i5 Processor M460 and $4 \mathrm{~Gb}$ of RAM. We used a collection of 30 benchmark bound constrained global optimization test problems of dimension 2-30, described in full detail in the Appendix B of [1]. Each problem is solved 30 times.

The population of $m=20$ fireflies is used and the algorithm stops when a maximum of 20000 function evaluations is reached. The parameters used are: $\beta_{0}=0.8$, $\gamma=1, \alpha=0.5$ and $d=1$.

In the first set of experiments, different norms in (2) are used for the calculation of the distance $r$. In the second set of experiments, a comparison with three attractiveness functions is made. For comparison purposes, performance profiles are used. Finally, a table with the results obtained with the best strategy is presented.

To compare the results obtained by different algorithms (or solvers), we used a statistical tool called performance profiles, created by Dolan and Moré [2]. Performance profiles are depicted by a plot of a cumulative distribution $\rho(\tau)$ representing a performance ratio for the different solvers, based on a chosen metric. Generally, performance profiles can be defined representing a statistic computed for a given quality indicator value obtained in several runs. Performance profiles provide a good visualization and easiness of making inferences about the performance of the algorithms. The concept of performance profiles requires minimization of a performance metric and can be described as follows.

Let $\wp$ and $\mathcal{S}$ be the set of problems and the set of solvers in comparison, respectively. Let $m_{p, s}$ be the performance metric found by solver $s \in \mathcal{S}$ on problem $p \in \wp$ after a fixed number of function evaluations. Here, a metric that measures the relative 
improvement of the function values, a scaled distance to the optimal function value, is defined by:

$$
m_{p, s}=\frac{f_{\text {avg } p, s}-f_{o p t}}{f_{\text {worst }}-f_{\text {opt }}}
$$

where $f_{\text {opt }}$ denotes the known optimal function value for a problem $p, f_{\text {worst }}$ denotes the worst function value found among all solvers on the problem $p$ and $f_{a v g_{p, s}}$ denotes the average of the best function values found by a solver $s$ on problem $p$, after a certain number of runs.

As $\min \left\{\mathrm{m}_{\mathrm{p}, \mathrm{s}}: \mathrm{s} \in \mathcal{S}\right\}$ can be zero for a particular problem, the comparison used in our study is based on the performance ratios defined by:

$$
r_{p, s}= \begin{cases}1+m_{p, s}-\min \left\{m_{p, s}: s \in \mathcal{S}\right\}, & \text { if } \min \left\{m_{p, s}: s \in \mathcal{S}\right\}<\mathcal{\varepsilon} \\ \frac{m_{p, s}}{\min \left\{m_{p, s}: s \in \mathcal{S}\right\}}, & \text { otherwise }\end{cases}
$$

for $p \in \wp, s \in \mathcal{S}$ and $\varepsilon=0.00001$.

The overall assessment of the performance of a particular solver $s$ is given by

$$
\rho_{s}(\tau)=\frac{\#\left\{p \in \wp: r_{p, s} \leq \tau\right\}}{\# \mathcal{S}}, \tau \in \mathbb{R} .
$$

The value of $\rho_{s}(1)$ gives the probability that the solver $s$ will win over the others in comparison. Thus, to see which solver has the least value of the performance metric mostly, then $\rho_{s}(1)$ should be compared for all the solvers. The higher the $\rho_{s}$ the better the solver is. On the other hand, for large values of $\tau$, the solver robustness is measured by $\rho_{s}(\tau)$.

Figure 1 plots the performance profile for the average value of obtained best function values concerning FA with different norms in the attractiveness function (2). From Fig. 1 we may conclude that the average best solutions found for computing attractiveness based on 1-norm, over the 30 runs, outperforms the other versions in comparison. In particular, this one gives the best average solution in about $50 \%$ of the tested problems and dominates the other four solvers for all $\tau$ values.

Figure 2 shows the plots of the solvers in comparison that are related with the implementation of the two attractiveness functions ((5) and (6)) and the original attractiveness function (2). In all attractiveness functions the 1-norm is considered to compute the distance between fireflies. The attractiveness function defined by $\beta^{1}$ reveals as efficient as the original beta function. Their efficiency is shown in Fig. 2 at $\tau=1$. However, for $\tau$ greater than approximately 2 , the solver with $\beta^{1}$ as the attractiveness function wins against the others and dominates with respect to robustness. 


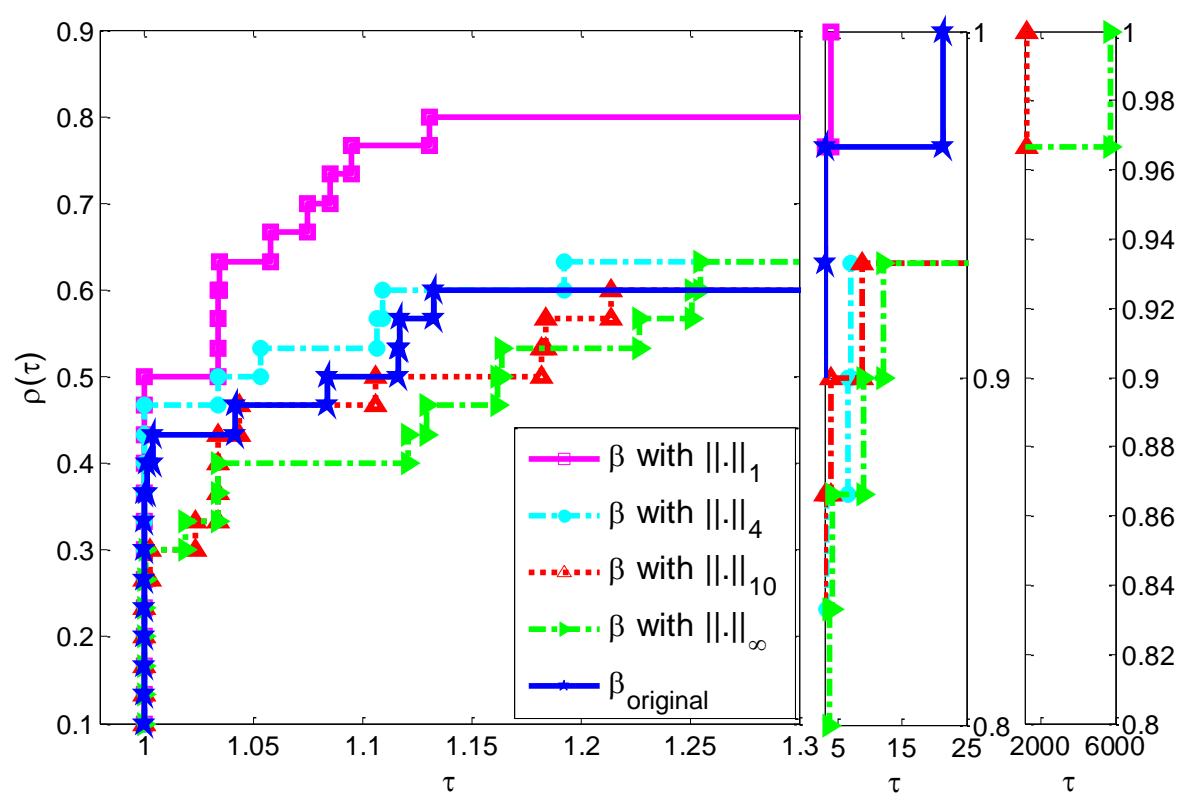

Fig. 1. Performance profiles on $f_{\text {avg }}$ with different norms for computing distance.

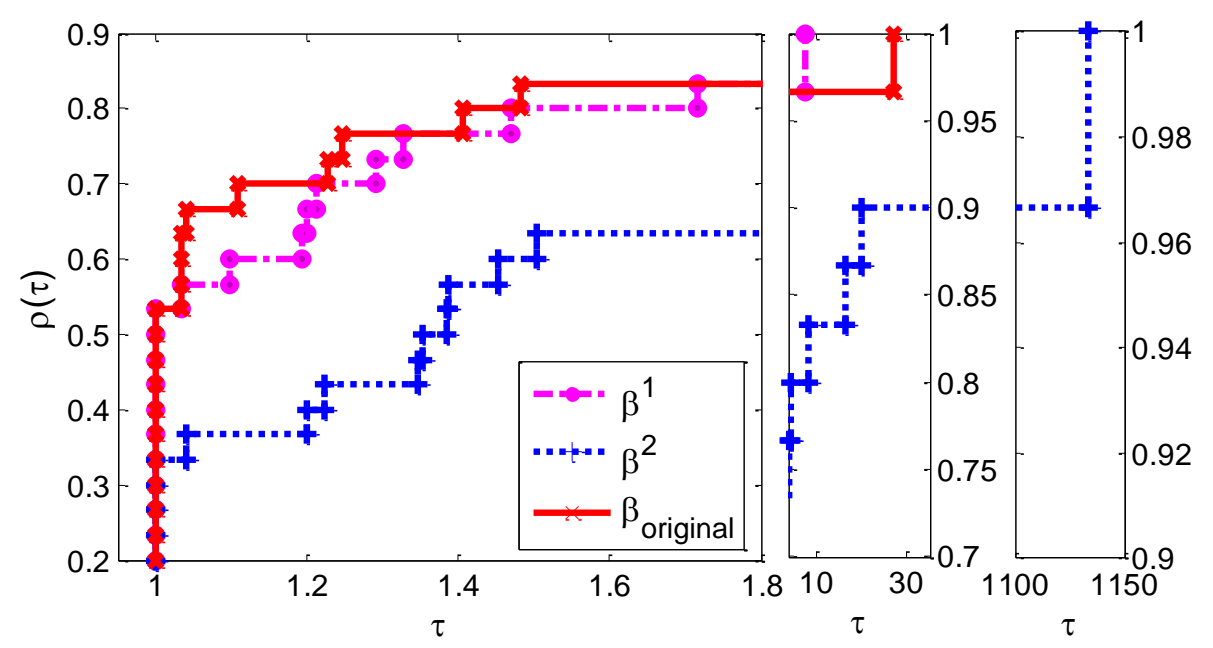

Fig. 2. Performance profiles on $f_{\text {avg }}$ with different attractiveness functions.

Table 1 summarizes some of the numerical results produced by version of FA with the attractiveness function $\beta^{1}$ and the distance $r$ computed by 1-norm, within 20000 function evaluations among 30 runs. The first column shows the problems names, 
followed by the dimension of the problem and in the third column the known global optimum $\left(f_{\text {opt }}\right)$. The following columns present: the best value $\left(f_{\text {best }}\right)$, the median $\left(f_{\text {med }}\right)$ and the worst value $\left(f_{\text {worst }}\right)$, and the standard deviation (SD), of obtained function values over the 30 runs.

Table 1. Results produced by FA using $\beta^{1}$, where $r$ is computed with $\|.\|_{1}$.

\begin{tabular}{|c|c|c|c|c|c|c|}
\hline Problem & $n$ & $f_{\text {opt }}$ & $f_{\text {best }}$ & $f_{\text {med }}$ & $f_{\text {worst }}$ & SD \\
\hline Ackley & 2 & 0.000000 & $1.965 \mathrm{E}-11$ & $8.375 \mathrm{E}-11$ & $1.925 \mathrm{E}-10$ & $3.98 \mathrm{E}-11$ \\
\hline Beale & 2 & 0.000000 & $2.112 \mathrm{E}-22$ & $3.330 \mathrm{E}-21$ & $2.105 \mathrm{E}-20$ & $6.10 \mathrm{E}-21$ \\
\hline Boh1 & 2 & 0.000000 & $0.000 \mathrm{E}+00$ & $0.000 \mathrm{E}+00$ & $0.000 \mathrm{E}+00$ & $0.00 \mathrm{E}+00$ \\
\hline Boh2 & 2 & 0.000000 & $0.000 \mathrm{E}+00$ & $0.000 \mathrm{E}+00$ & $1.110 \mathrm{E}-16$ & $3.15 \mathrm{E}-17$ \\
\hline Boh3 & 2 & 0.000000 & $0.000 \mathrm{E}+00$ & $0.000 \mathrm{E}+00$ & $5.551 \mathrm{E}-17$ & $1.69 \mathrm{E}-17$ \\
\hline Booth & 2 & 0.000000 & $4.682 \mathrm{E}-22$ & $1.916 \mathrm{E}-20$ & $2.078 \mathrm{E}-19$ & $4.11 \mathrm{E}-20$ \\
\hline Branin & 2 & 0.397887 & 0.397887 & 3.979E-01 & $3.979 \mathrm{E}-01$ & $2.26 \mathrm{E}-16$ \\
\hline Dixon-price & 2 & 0.000000 & $1.114 \mathrm{E}-22$ & $2.736 \mathrm{E}-20$ & $2.149 \mathrm{E}-19$ & $5.36 \mathrm{E}-20$ \\
\hline Easom & 2 & -1.000000 & -1.000000 & -1.000000 & $0.000 \mathrm{E}+00$ & $3.79 \mathrm{E}-01$ \\
\hline Golstein-Price & 2 & 3.000000 & 3.000000 & 3.000000 & 3.000000 & $0.00 \mathrm{E}+00$ \\
\hline Griewank & 2 & 0.000000 & $0.000 \mathrm{E}+00$ & 0.007396 & 0.027125 & $5.84 \mathrm{E}-03$ \\
\hline Hartman3 & 3 & -3.862782 & -3.862782 & -3.862782 & -3.861768 & $2.39 \mathrm{E}-04$ \\
\hline Hartman6 & 6 & -3.322368 & -3.322368 & -3.322368 & -3.141611 & $5.82 \mathrm{E}-02$ \\
\hline Hump & 2 & -1.031629 & -1.031628 & -1.031628 & -1.031628 & $4.52 \mathrm{E}-16$ \\
\hline Levy & 30 & 0.000000 & 0.020419 & 0.233172 & 1.243473 & $2.80 \mathrm{E}-01$ \\
\hline Matyas & 2 & 0.000000 & $6.655 \mathrm{E}-24$ & $5.949 \mathrm{E}-22$ & $6.379 \mathrm{E}-21$ & $1.21 \mathrm{E}-21$ \\
\hline Mich & 2 & -1.801300 & -1.801303 & -1.801303 & -1.801303 & $6.78 \mathrm{E}-16$ \\
\hline Perm & 4 & 0.000000 & 0.022767 & 5.195744 & 101.536442 & $2.02 \mathrm{E}+01$ \\
\hline Powell & 24 & 0.000000 & 0.689417 & 2.469367 & 11.843144 & $2.71 \mathrm{E}+00$ \\
\hline Power & 4 & 0.000000 & 0.000255 & 0.009421 & 0.092230 & $2.80 \mathrm{E}-02$ \\
\hline Rastrigin & 2 & 0.000000 & $0.000 \mathrm{E}+00$ & $0.000 \mathrm{E}+00$ & $0.000 \mathrm{E}+00$ & $0.00 \mathrm{E}+00$ \\
\hline Rosenbrock & 2 & 0.000000 & $8.124 \mathrm{E}-21$ & $2.284 \mathrm{E}-18$ & $2.469 \mathrm{E}-04$ & $5.87 \mathrm{E}-05$ \\
\hline Shekel5 & 4 & -10.153200 & -10.153200 & -10.153200 & -2.682860 & $2.58 \mathrm{E}+00$ \\
\hline Shekel7 & 4 & -10.402941 & -10.402941 & -10.402941 & -10.402941 & $5.42 \mathrm{E}-15$ \\
\hline Shekel10 & 4 & -10.536410 & -10.536410 & -10.536410 & -2.427335 & $1.48 \mathrm{E}+00$ \\
\hline Shubert & 2 & -186.730909 & -186.730909 & -186.730909 & -180.251858 & $1.19 \mathrm{E}+00$ \\
\hline Sphere_30 & 30 & 0.000000 & 0.000964 & 0.002590 & 0.003891 & 8.07E-04 \\
\hline Sum-squares & 20 & 0.000000 & 0.013425 & 0.360771 & 3.942155 & $1.06 \mathrm{E}+00$ \\
\hline Trid & 10 & -200.000000 & -208.708837 & -174.090109 & -95.873874 & $2.91 \mathrm{E}+01$ \\
\hline Zakharov & 2 & 0.000000 & $2.773 \mathrm{E}-23$ & $3.904 \mathrm{E}-21$ & $1.818 \mathrm{E}-20$ & $5.35 \mathrm{E}-21$ \\
\hline
\end{tabular}


The computed solutions are of high quality and the obtained $f_{\text {best }}$ solutions obtained for the problems are very close to the known minimum, except for Perm, Powell, Power, Sphere_30, Sum_squares and Trid problems. The values of SD are in general, for all problems, reasonably small showing the consistency of this variant of FA algorithm.

\section{Conclusions}

In this paper the Firefly Algorithm, a stochastic global optimization algorithm, inspired by the social behavior of fireflies and based on their flashing and attraction, is presented for solving the bound constrained optimization problems.

In order to improve the efficiency of FA, other ways of calculating the distance between the points and other functions to compute the attractiveness of fireflies were tested and analyzed.

A set of benchmark global optimization test problems were used to show the performance of the proposed modified FAs and preliminary results revealed competitive when comparing with the original FA version.

In the future, an extension of FA algorithm, to solve constrained problems, based on penalty techniques will be addressed.

Acknowledgements. This work has been supported by FCT (Fundação para a Ciência e Tecnologia, Portugal) in the scope of the projects: PEst-OE/MAT/UI0013/2014 and PEst-OE/EEI/UI0319/2014.

\section{References}

1. Ali, M.M., Khompatraporn, C., Zabinsky, Z.B.: A numerical evaluation of several stochastic algorithms on selected continuous global optimization test problems. J. Global Optim. 31, 635-672 (2005)

2. Dolan, E. D., Doré, J.J.: Benchmarking Optimization Software with Performance Profiles. Preprint ANL/MCS-P861-1200 (2001)

3. Dorigo, M., Stützle, T.: Ant Colony Optimization, MIT Press (2004)

4. Dorigo, M., Caro, G. D., Gambardella L. M.: Ant algorithms for discrete optimization,. Université Libre de Bruxelles, Belgium (1999)

5. Eberhart, R.C., Kennedy, J., Shi, Y.: Swarm optimization. Academic Press (2001)

6. Eberhart, R.C., Kennedy, J.: Particle Swarm optimization. Proc. of IEEE International Conference on Neural Networks, Piscataway, NJ, pp.1942-1948 (1995)

7. Goldber, D.E.: Genetic Algorithms in Search, Optimization and Machine Learning. Reading, Mass., Addison Wesley (1989)

8. Heppner, F., Grenander, U.: A stochastic nonlinear model for coordinated bird flocks. The Ubiquity of Chaos. AAAS Publications, Washington DC (1990) 
9. Lukasik, S., Zak, S.: Firefly algorithm for continuous constrained optimization tasks. In: Chen, S.M., Ngugen, N.T., Kowalczyk, R. (eds.), ICCC 2009, Lecture notes in Artificial Intelligence, vol. 5796, pp. 97-100. Springer (2009)

10. Reynolds, C. W.: Flocks, herds and schools: a distributed behavioral model. Comp. Graph. 25-34 (1987)

11. Rocha, A.M.C., Fernandes, E.M.G.P., Martins, T.F.M.C.: Novel Fish swarm heuristics for bound constrained global optimization problems. J. Comput. Appl. Math. 235(16), 4611$4620(2011)$

12. Yang, X-S.: Firefly Algorithm, Stochastic Test Functions and Design Optimization. Int. J. Bio-Inspired Computation, Vol. 2, No. 2, pp.78-84 (2010)

13. Yang, X-S.: Nature-Inspired Metaheuristic Algorithms. Luniver Press, Beckington, UK, 2nd edition, 2010

14. Yang, X-S.: Firefly algorithms for multimodal optimization. In: Watanabe O, Zeugmann T, (eds.) Stochastic algorithms: foundations and applications, SAGA 2009, LNCS, vol. 5792, pp. 169-78. Springer-Verlag (2009) 